\title{
Nonadiabatic geometric quantum computation with trapped ions
}

\author{
Xin-Qi Li, ${ }^{1,2,3}$ Li-Xiang Cen, ${ }^{1}$ Guoxiang Huang, ${ }^{2}$ Lei Ma, ${ }^{2}$ and YiJing Yan $^{3}$ \\ ${ }^{1}$ National Laboratory for Superlattices and Microstructures, Institute of Semiconductors, Chinese Academy of Sciences, P.O. Box 912, \\ Beijing 100083, China \\ ${ }^{2}$ Key Laboratory for Optical and Magnetic Resonance Spectroscopy, and Department of Physics, East China Normal University, \\ Shanghai 200062, China \\ ${ }^{3}$ Department of Chemistry, Hong Kong University of Science and Technology, Kowloon, Hong Kong
}

(Received 10 April 2002; published 23 October 2002)

\begin{abstract}
We propose a nonadiabatic scheme for geometric quantum computation with trapped ions. By making use of the Aharonov-Anandan phase, the proposed scheme not only preserves the globally geometric nature in quantum computation, but also provides the advantage of nonadiabaticity that overcomes the problem of slow evolution in the existing adiabatic schemes. Moreover, the present scheme requires only two atomic levels in each ion, making it an appealing candidate for quantum computation.
\end{abstract}

DOI: 10.1103/PhysRevA.66.042320

PACS number(s): 03.67.Lx, 73.61.-r, 42.50.Vk

Conventionally, the controllable operations in quantum computation (QC) are achieved on the basis of dynamical origins of quantum state evolutions [1]. In recent years, for the purpose of being fault tolerant to certain types of computational errors, there are considerable interests in exploiting the possibility of implementing quantum computation by geometrical means which have been termed as holonomic quantum computations [2-4]. Depending on the degenerate property of the eigenspace of the governing Hamiltonian, the holonomy can be either a simple Abelian Berry phase factor [5] or a general non-Abelian unitary transformation [6]. It has been shown that the universal quantum computation can be implemented in principle by holonomies [2-4]. Up to date, several experimental proposals have been suggested for geometric quantum computation, using such as the nuclear magnetic resonance (NMR) [7], superconducting nanocircuits $[8,9]$, trapped ions [10,11], and nonlinear optics [12].

The principle of the aforementioned geometric QC is rooted in the adiabatic evolution of the quantum system, which may thus imply a slow computing speed. The adiabatically slow evolution may also challenge the sustainment of the required coherence in QC. Therefore, geometric QC based on nonadiabatic evolution should be desirable. Very recently, Wang and Keiji suggested to exploit a nonadiabatic evolution to realize geometric QC in NMR system and superconductor nanocircuits [13]. Indeed, geometric phase exists in nonadiabatic evolving quantum systems, which is in fact the Aharonov-Anandan (AA) phase [14]. Strictly speaking, the AA phase depends on certain dynamical quantities such as the rotating angular speed of external (magnetic) field or state vector [16-18]. In this sense, the AA phase differs from the adiabatic Berry phase. However, the dependence of the AA phase on the angular speed is through the closed path loop depicted by the ending point of the state vector, and hence is global in nature that largely retains the geometric sense of the AA phase. Accordingly, quantum computation based on the nonadiabatic AA phase can be reasonably regarded as a kind of geometric QC.

In this work we propose a scheme for nonadiabatic geometric quantum computation with trapped ions. Besides removing the drawback of the slow adiabatic evolution, the proposed nonadiabatic scheme holds additional merits as follows. First, there is no need to design the reverse evolving path to eliminate dynamical phases that occur in the Berry phase-based, adiabatic geometric QC operations in nondegenerated systems $[7,8]$. Second, in comparison to the existing fully holonomic QC schemes $[9,10]$, the present one does not involve the complicated construction of the degenerate eigenspace of driving Hamiltonian. The above two merits stem from the following observations. In a nonadiabatic quantum evolution, the geometric AA phase is in general accompanied by a dynamical phase. However, if the evolving path is designed such that along it the state vector is always perpendicular to the driving (magnetic) field, the resulting phase factor after a nontrivial cyclic evolution will be purely geometric. This feature has been exploited by Suter et al. in their seminal experiment for demonstrating the AA phase [15]. Finally, only two atomic levels of each ion are needed in our scheme. This merit alone is attractive, since the originally proposed ion-trap QC scheme required three levels [19] and the recently proposed holonomic ion-trap QC required four levels $[10,11]$. We notice that valuable efforts on improving ion-trap QC protocol by using only two levels have been carried out in dynamic schemes [20-22]. In particular, the technique proposed in Ref. [22], which effectively couples the electronic states of a pair of ions via virtually exchanging phonons, is similar to our present one. The major contribution of our work is to perform $\mathrm{QC}$ by geometric means. Viewing that the work of Ref. [22] has in fact extended the ion-trap QC scheme to finite temperature, our geometric scheme may also hold to similar regime, although in the following we would restrict our description at zerotemperature limit.

Model description. For quantum logic with trapped ions, we assume that each ion has two relevant internal states $|0\rangle$ and $|1\rangle$ with energy separation $\omega_{0}$, and, as usual [19], can be selectively addressed by lasers. Consider, for instance, the $j$ th ion being exposed to a traveling-wave laser field $\mathbf{E}(\mathbf{z})$ $=\mathbf{E}_{0} \cos \left(\mathbf{k} \cdot \mathbf{z}-\omega_{L} t+\phi\right)$ with frequency $\omega_{L}$, wave vector $\mathbf{k}$, and phase $\phi$. Here $\mathbf{z}=z_{0} \hat{\mathbf{z}}\left(a+a^{\dagger}\right)$ is the center-of-mass coordinate of the ion in terms of the phonon raising (lowering) 
operator $a^{\dagger}(a)$ and zero-point spread $z_{0} \equiv(\hbar / 2 M \omega)^{1 / 2}$, where $M$ is the total mass of the ion chain, and $\omega$ is the phonon frequency. The resulting Hamiltonian reads

$$
\mathcal{H}^{(j)}=\frac{\omega_{0}}{2} \sigma_{j}^{z}+\tilde{\omega}_{j}\left[\sigma_{j}^{+} e^{i \eta\left(a+a^{\dagger}\right)-i \omega_{L} t+i \phi}+\text { H.c. }\right] .
$$

Here, the atomic Pauli operators $\sigma^{z}=|1\rangle\langle 1|-| 0\rangle\langle 0|, \sigma^{+}$ $=|1\rangle\langle 0|$, and $\sigma^{-}=|0\rangle\langle 1|$, are introduced. In Eq. (1) $\widetilde{\omega}_{j}$ is the Rabi frequency, and $\eta=(\mathbf{k} \cdot \hat{\mathbf{z}}) z_{0}$ is the Lamb-Dicke parameter that accounts for the coupling strength between internal and motional states. In our geometric scheme, the phonon plays no roles in one-bit operation, but it does involve in two-bit logic gate implementation as it effectively couples two ions together.

One-bit rotation via nonadiabatic AA phase. As just mentioned, in single-bit logic operation, we do not need the phonon-assisted dynamical flipping. This can be the case under situations such as (i) at zero temperature (no phonon excitation), if $\omega_{L}<\omega_{0}$; and (ii) at finite temperature, but $\omega_{0}-\omega_{L} \neq n \omega$ ( $n$ is an integer). In the absence of phonon participation, Eq. (1) can be reexpressed as

$$
\mathcal{H}_{R}^{(j)}=\boldsymbol{\Omega}_{j} \cdot \boldsymbol{\sigma}_{j},
$$

in the rotating frame (with angular velocity $\omega_{L} \hat{\mathbf{e}}_{z}$ ). Here notations have been introduced for the effective magnetic field $\boldsymbol{\Omega}_{j} \equiv\left\{\tilde{\omega}_{j} \cos \phi, \tilde{\omega}_{j} \sin \phi,\left(\omega_{0}-\omega_{L}\right) / 2\right\}$, and the vector Pauli operator $\boldsymbol{\sigma}_{j} \equiv\left\{\sigma_{j}^{x}, \sigma_{j}^{y}, \sigma_{j}^{z}\right\}$.

To show how rotation between logic states $|0\rangle$ and $|1\rangle$ can be performed by geometric means, we first consider the geometric evolution of the eigenstates of $\sigma^{y}$, defined by $\sigma^{y}| \pm\rangle= \pm| \pm\rangle$, under appropriately designed laser sequences. Specifically, the state $|+\rangle$, for instance, will complete a cyclic evolution and acquire a nonadiabatic AA phase under the following operations:

(i) Switching on a $\pi$ pulse with laser phase $\phi=0$, the state $|+\rangle$ rotates around an effective magnetic field $\boldsymbol{\Omega}_{1}$ $=\left\{\tilde{\omega}_{j}, 0,\left(\omega_{0}-\omega_{L}\right) / 2\right\}$, from $|+\rangle$ in the $\hat{\mathbf{e}}_{y}$ direction to $|-\rangle$ in the $-\hat{\mathbf{e}}_{y}$ direction along the curve ACB on the Bloch sphere; see Fig. 1.

(ii) Suddenly changing the laser phase to $\phi=\pi$, after another $\pi$ pulse, the state $|-\rangle$ rotates back to $|+\rangle$ around $\boldsymbol{\Omega}_{2}$ $=\left\{-\tilde{\omega}_{j}, 0,\left(\omega_{0}-\omega_{L}\right) / 2\right\}$ along the curve BDA on the Bloch sphere.

According to the AA phase theory, after the above cyclic evolution, the state $|+\rangle$ will acquire a geometric phase $e^{i \gamma}$, with $\gamma=4 \arctan \left[2 \tilde{\omega}_{j} /\left(\omega_{0}-\omega_{L}\right)\right]$. Note that during the above operation, the state vector keeps always perpendicular to the effective magnetic field, thus no dynamical phase is accumulated in the evolution. Similarly, the state $|-\rangle$ will acquire AA phase $e^{-i \gamma}$ at the same time.

Now consider the evolution of logic states $|0\rangle$ $=-i / \sqrt{2}(|+\rangle-|-\rangle)$, and $|1\rangle=1 / \sqrt{2}(|+\rangle+|-\rangle)$. After the above operations, they evolve to states

$$
|0\rangle \rightarrow \cos \gamma|0\rangle+\sin \gamma|1\rangle,
$$

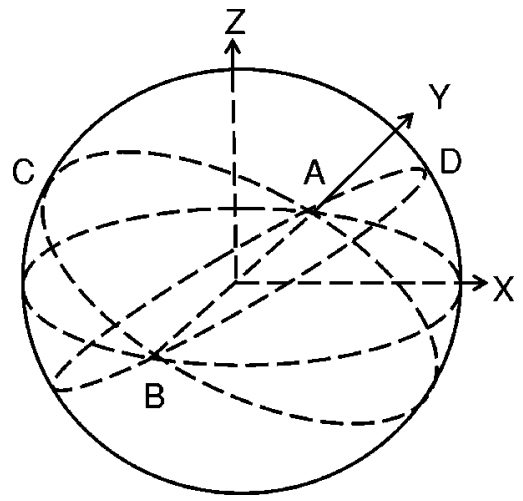

FIG. 1. Schematic diagram for geometric rotation of the state vector around the effective magnetic field. Since the state is always perpendicular to the field, there is no dynamical phase accumulation during the evolution.

$$
|1\rangle \rightarrow \cos \gamma|1\rangle-\sin \gamma|0\rangle .
$$

We see here that the geometric AA phase plays a role of rotating the logic states. Particularly, complete state flipping between $|0\rangle$ and $|1\rangle$ can take place at $\gamma=\pi / 2$. Note also that the possible value of $\gamma$ ranges from 0 to $2 \pi$, implying the ability of arbitrary rotation between $|0\rangle$ and $|1\rangle$.

The state evolution of the performed qubit described by Eq. (3) is expressed in the rotating frame with frequency $\omega_{L}$, in which other free (not performed) qubits would have relative phase accumulations in the nonresonant case of $\omega_{L}$ $\neq \omega_{0}$. Conventionally, a more convenient choice is to express states in the interaction picture with respect to $\mathcal{H}_{0}$ $=\omega_{0} / 2 \Sigma_{j} \sigma_{j}^{z}$ (equivalently, a rotating frame with frequency $\omega_{0}$ around $z$ axis). Accordingly, Eq. (3) can be recast in the interaction picture as

$$
\begin{aligned}
& |0\rangle \rightarrow e^{-i \omega_{D} \tau / 2} \cos \gamma|0\rangle+e^{i \omega_{D} \tau / 2} \sin \gamma|1\rangle, \\
& |1\rangle \rightarrow e^{i \omega_{D} \tau / 2} \cos \gamma|1\rangle-e^{-i \omega_{D} \tau / 2} \sin \gamma|0\rangle,
\end{aligned}
$$

where $\omega_{D}=\omega_{0}-\omega_{L}$ and $\tau$ is the total operation time on the performed qubit.

Interestingly, the above state rotation (flipping) is performed by nonresonant pulses via geometrical means. Now we show that by resonant pulses, a pure phase-shift gate of single qubit can be geometrically realized. In resonant case, the laser-frequency-associated rotating frame coincides with the interaction picture defined by $\mathcal{H}_{0}$, in which the $(j$ th) qubit Hamiltonian reads $\mathcal{H}^{(j)}=\widetilde{\omega}_{j}\left[\sigma_{j}^{+} e^{i \phi}+\right.$ H.c. $]=\boldsymbol{\Omega}_{j} \cdot \boldsymbol{\sigma}_{j}$, with $\boldsymbol{\Omega}_{j}=\left\{\tilde{\omega}_{j} \cos \phi, \tilde{\omega}_{j} \sin \phi, 0\right\}$. We see that in the rotating frame the effective magnetic field constantly has zero $z$-axis component for arbitrary laser phase $\phi$, i.e., it lies in the $x-y$ plane. To realize the single-bit phase gate, we first switch on a $\pi$ pulse with laser phase at certain value, say, $-\phi_{0}$. The logic state $|0\rangle$ and $|1\rangle$ would rotate around the effective magnetic field $\left\{\tilde{\omega}_{j} \cos \phi_{0},-\tilde{\omega}_{j} \sin \phi_{0}, 0\right\}$ to $|1\rangle$ and $|0\rangle$, respectively. Then, suddenly changing the laser phase to $\phi_{0}$, after another $\pi$ pulse, the logic states rotate around the effective magnetic field $\left\{\tilde{\omega}_{j} \cos \phi_{0}, \tilde{\omega}_{j} \sin \phi_{0}, 0\right\}$, and return to 
the original states $|0\rangle$ and $|1\rangle$. Associated with this two-step cyclic evolution, the logic states will, respectively, acquire geometric AA phases as

$$
\begin{gathered}
|0\rangle \rightarrow e^{i \tilde{\gamma}}|0\rangle, \\
|1\rangle \rightarrow e^{-i \tilde{\gamma}}|1\rangle,
\end{gathered}
$$

where $\tilde{\gamma}=4 \phi_{0}$. With the help of this phase-shift operation, the additional phase factor in Eq. (4) can be canceled out by properly choosing $\phi_{0}$. More importantly, together with this phase-shift gate, the qubit state rotation Eq. (4) constitutes a complete logic set for the arbitrary single-qubit operation.

Two-bit gate via nonadiabatic AA phase. To complete the universal gate for quantum computation, nontrivial two-bit gate such as the CNOT, or equivalently, the conditional phaseshift (CPS) gate, would be required. Below we show how the CPS gate can be implemented via geometric means. Consider two qubits (e.g., the $j$ th and $k$ th ones) irradiated by two lasers with frequencies $\omega_{L, 1}$ and $\omega_{L, 2}$, and phases $\phi_{1}$ and $\phi_{2}$. By setting $\omega_{L, 1}>\omega_{0}$ and $\omega_{L, 2}<\omega_{0}$, and correspondingly denoting the detunings by $\delta_{1}=\omega_{L, 1}-\omega_{0}$ and $\delta_{2}=\omega_{0}$ $-\omega_{L, 2}$, the effective coupling between the two-bit states $|00\rangle$ and $|11\rangle$ can be established via virtually exchanging phonons, and the resulting two-bit effective Hamiltonian reads [22]

$$
\begin{aligned}
\widetilde{\mathcal{H}}^{(j, k)}= & \sum_{m=1}^{4} E_{m}|\tilde{m}\rangle\langle\tilde{m}|+g_{j k}\left[e^{-i\left(\omega_{L, 1}+\omega_{L, 2}\right) t} e^{i\left(\phi_{1}+\phi_{2}\right)} \sigma_{j}^{+} \sigma_{k}^{+}\right. \\
& + \text {H.c. }] .
\end{aligned}
$$

Here, notations $\{|\widetilde{1}\rangle=|11\rangle,|\widetilde{2}\rangle=|00\rangle,|\widetilde{3}\rangle=|10\rangle,|\widetilde{4}\rangle=|01\rangle\}$ are introduced for the two-bit computational basis states. Up to the first-order expansion of the Lamb-Dicke parameter $\eta$ in Eq. (1), the effective coupling strength can be obtained via second-order perturbation theory as $g_{j k}=g_{j} g_{k}\left[1 /\left(\delta_{1}-\omega\right)\right.$ $\left.-1 /\left(\delta_{2}+\omega\right)\right]$, where $g_{j(k)}=\tilde{\omega}_{j(k)} \eta$ is the one-phonon involved Rabi frequency of single-ion transition. The four basis-state energies $E_{m}(m=1, \ldots, 4)$ contain also the ac Stark shifts. The effective interaction couples only between $|\widetilde{1}\rangle$ and $|\widetilde{2}\rangle$, but leaves $|\widetilde{3}\rangle$ and $|\widetilde{4}\rangle$ inactive with respect to the laser operation in study. This effective two-state dynamics (e.g., Rabi oscillations) has been demonstrated in Ref. [22] by numerical simulation starting from the original Hamiltonian. As a result, for two-bit operation, one can focus on state evolution in the subspace $\{|\widetilde{1}\rangle,|\widetilde{2}\rangle\}$ in which the two-bit Hamiltonian (6) is recast to a similar form as the single qubit [23]

$$
\widetilde{\mathcal{H}}_{R}^{(j, k)}=\frac{\tilde{\omega}_{D}}{2} \Sigma^{z}+g_{j k}\left(e^{i \Phi} \Sigma^{+}+e^{-i \Phi} \Sigma^{-}\right),
$$

where $\Phi=\phi_{1}+\phi_{2}$, and the two-bit Pauli matrices are introduced as $\Sigma^{z}|\widetilde{1}(\widetilde{2})\rangle= \pm|\widetilde{1}(\widetilde{2})\rangle, \quad \Sigma^{+}|\widetilde{2}\rangle=|\widetilde{1}\rangle$, and $\Sigma^{-}|\widetilde{1}\rangle$ $=|\widetilde{2}\rangle$. Note also that the Hamiltonian (7) has been expressed in the rotating frame with respect to $\Sigma^{z}$ with the rotation frequency $\omega_{L, 1}+\omega_{L, 2}$, thus $\tilde{\omega}_{D}=\left(E_{1}-E_{2}\right)-\left(\omega_{L, 1}+\omega_{L, 2}\right)$. Simple comparison of Eq. (7) with Eq. (2) indicates that an arbitrary rotation between $|\widetilde{1}\rangle$ and $|\widetilde{2}\rangle$ can be performed geometrically as that in the single-bit case.

In the spirit of pulse-sequence operations in dynamic scheme based on an $X Y$ spin model [24-26], we shall in the following show that the CPS gate can be implemented geometrically as

$$
\begin{aligned}
\mathcal{U}_{\mathrm{CPS}}= & e^{i \pi / 4} e^{i \pi \mathbf{n}_{j} \cdot \boldsymbol{\sigma}_{j} / 3} e^{i \pi \mathbf{n}_{k} \cdot \boldsymbol{\sigma}_{k} / 3} e^{-i \pi \sigma_{k}^{x} / 2} U_{j k}(\pi / 4) e^{-i \pi \sigma_{j}^{y} / 2} \\
& \times U_{j k}(\pi / 4) e^{-i \pi \sigma_{j}^{x} / 2} .
\end{aligned}
$$

Here the vector Pauli operator $\boldsymbol{\sigma}=\left(\sigma^{x}, \sigma^{y}, \sigma^{z}\right)$, unit vector $\mathbf{n}_{j}=(1,1,-1) / \sqrt{3}$, and $\mathbf{n}_{k}=(1,-1,1) / \sqrt{3}$. In the interaction picture with respect to the original free ion Hamiltonian, the two-bit rotation operator $U_{j k}(\alpha)$ is defined via $U_{j k}(\alpha)|01\rangle$ $=|01\rangle, U_{j k}(\alpha)|10\rangle=|10\rangle$, and

$$
\begin{aligned}
& U_{j k}(\alpha)|00\rangle=\cos \left(\frac{\alpha}{2}\right)|00\rangle+i \sin \left(\frac{\alpha}{2}\right)|11\rangle, \\
& U_{j k}(\alpha)|11\rangle=\cos \left(\frac{\alpha}{2}\right)|11\rangle+i \sin \left(\frac{\alpha}{2}\right)|00\rangle .
\end{aligned}
$$

Since the arbitrary rotation and phase-shift gate of single qubit have been built up, the single-bit operations in Eq. (8) can be readily implemented via geometric means by properly combining the single-bit logic elements. Viewing the similarity between the two-bit reduced Hamiltonian (7) in the subspace $\{|00\rangle,|11\rangle\}$ and the single-bit Hamiltonian (2), the two-bit rotation of Eq. (9) can be straightforwardly implemented by the following two-step procedures:

(i) In the two-bit rotating frame with frequency $\omega_{L, 1}$ $+\omega_{L, 2}$ around $\Sigma^{z}$, performing cyclic evolution for the eigenstates of $\Sigma^{y}$ by controlling the laser phases $\Phi$ similarly as in the single-bit case, one can geometrically rotate the states $|00\rangle$ and $|11\rangle$. Expressed in the interaction picture which also corresponds to $\widetilde{\mathcal{H}}_{0} \equiv E_{1}|11\rangle\left\langle 11\left|+E_{2}\right| 00\right\rangle\langle 00|$, this operation can realize the following state transformation:

$$
\begin{gathered}
|00\rangle \rightarrow e^{-i \tilde{\omega}_{D} \tau / 2} \cos \Gamma|00\rangle+e^{i \tilde{\omega}_{D} \tau / 2} \sin \Gamma|11\rangle, \\
|11\rangle \rightarrow e^{i \tilde{\omega}_{D} \tau / 2} \cos \Gamma|11\rangle-e^{-i \tilde{\omega}_{D} \tau / 2} \sin \Gamma|00\rangle .
\end{gathered}
$$

Here $\Gamma$ is the geometric AA phase determined by the evolution contour of the two-bit state vector, and $\tilde{\omega}_{D} \tau$ is the detuning-induced phase accumulation.

(ii) Tuning the laser frequencies in resonance with the two ions [27], i.e., $\tilde{\omega}_{D}=0$, a phase-shift gate associating with $|00\rangle$ and $|11\rangle$ can be implemented to cancel out the phase factors in Eq. (10). In the resonance case the $\omega_{L, 1}+\omega_{L, 2}$ rotating frame coincides with the interaction picture of $\widetilde{\mathcal{H}}_{0}$. As the one-bit resonant case, the effective magnetic field corresponding to Eq. (7) now lies in the $x-y$ plane since $\tilde{\omega}_{D}=0$. By successively choosing two different values of the laser phase $\Phi$, one can perform two $\pi$ rotations on the states $|11\rangle$ and $|00\rangle$ around the effective magnetic fields, and 
readily generate the AA geometric phases, $e^{-i \widetilde{\Gamma}}$ and $e^{i \widetilde{\Gamma}}$, for the states $|11\rangle$ and $|00\rangle$, respectively. Now, after a phaseshift operation with $\widetilde{\Gamma}=\tilde{\omega}_{D} \tau / 2-\pi / 4$, Eq. (10) becomes,

$$
\begin{gathered}
|00\rangle \rightarrow e^{-i \pi / 4}[\cos \Gamma|00\rangle+i \sin \Gamma|11\rangle], \\
|11\rangle \rightarrow e^{i \pi / 4}[\cos \Gamma|11\rangle+i \sin \Gamma|00\rangle] .
\end{gathered}
$$

This is identical to Eq. (9), except for the additional global phases. Obviously, these global phases Quite simply, the global phases in Eq. (9) will not appear if we first generate a phase shift of $e^{i \pi / 4}$ on $|00\rangle$ and $e^{-i \pi / 4}$ on $|11\rangle$ at the same time, by the phase-shift gate just described, prior to the operation of Eq. (10).

We have thus realized the two-bit gate $U_{j k}$ as defined in Eq. (9). Together with the arbitrary one-bit operations (rotation and phase shift) described earlier, we can now readily implement the important CPS gate Eq. (8), whose role is to transform $|11\rangle \rightarrow e^{i \pi}|11\rangle$, while to keep other computational two-bit basis states unchanged.

Conclusion and discussion. The proposed nonadiabatic geometric QC scheme based on the AA phases is expected to overcome several drawbacks of the adiabatic schemes, namely, the slow evolution, need of refocusing to eliminate the dynamical phases, and continuous control over many fields to construct nontrivial loops in the parameter space. Viewing that the trapped ion is one of the best exploited systems for quantum computation, and that the proposed scheme requires a relatively simple atomic level configuration, we suggest, as a first step, to exploit it as an interferometer for principle proof of the nonadiabatic geometric AA phase discussed in this work. We believe that the interference associated with the nonadiabatic AA phase can be readily demonstrated by experiment in ion-trap systems.

As a possible QC architecture, the elementary operation steps in the proposed nonadiabatic geometric scheme are comparable to its dynamic counterparts. Specifically, the time scales for both the one-bit and two-bit geometric operations are about the same as those in the dynamic operations. Concerning the possible fault tolerance, in the adiabatic case, quantum logic is tolerant to certain types of errors such as the field fluctuations that preserve the loop area in parameter space, i.e., the Berry phase. Similarly, in the nonadiabatic case, the AA phase is of error tolerance to any fluctuation around the state-evolving path that preserves the path loop area. In principle, there exist many possible driving field deviations that can preserve the state path loop area. However, the most natural and possible errors appear to be random (but small) fluctuations of the laser phase, frequency, and coupling strength to the atomic levels, which equivalently result in fluctuations of the effective magnetic field. The global AA phase is expected to be largely immune from these types of errors, and, at the same time, the net dynamic phase accumulation is approximately zero due to the cancellation of the positive and negative contributions.

Support from the Major State Basic Research Project No. G001CB3095 of China, Special Fund for the "100 Person Project" from the Chinese Academy of Sciences, the Youth Science and Technology "Qimingxing" Program of Shanghai (No. 99QA14036), and the Research Grants Council of the Hong Kong Government is gratefully acknowledged.
[1] D. P. DiVincenzo, Science 269, 255 (1995); A. Ekert and R. Josza, Rev. Mod. Phys. 68, 733 (1996); A. M. Steane, Rep. Prog. Phys. 61, 117 (1998).

[2] P. Zanardi and M. Rasetti, Phys. Lett. A 264, 94 (1999).

[3] J. Pachos, P. Zanardi, and M. Rasetti, Phys. Rev. A 61, 010305(R) (2000).

[4] D. Ellinas and J. Pachos, Phys. Rev. A 64, 022310 (2001).

[5] M. V. Berry, Proc. R. Soc. London, Ser. A 392, 45 (1984).

[6] F. Wilczek and A. Zee, Phys. Rev. Lett. 52, 2111 (1984).

[7] J. A. Jones, V. Vedral, A. Ekert, and G. Castagnoli, Nature (London) 403, 869 (2000).

[8] G. Falci, R. Fazio, G. M. Palma, J. Siewert, and V. Vedral, Nature (London) 407, 355 (2000).

[9] M. S. Choi, e-print quant-ph/0111019.

[10] L. M. Duan, J. I. Cirac, and P. Zoller, Science 292, 1695 (2001).

[11] J. Pachos and H. Walther, e-print quant-ph/0111088, Phys. Rev. Lett. (to be published).

[12] J. Pachos and S. Chountasis, Phys. Rev. A 62, 052318 (2000).

[13] X. B. Wang and M. Keiji, Phys. Rev. B 65, 172508 (2002); quant-ph/0105024.

[14] Y. Aharonov and J. Anandan, Phys. Rev. Lett. 58, 1593 (1987).

[15] D. Suter, K. T. Mueller, and A. Pines, Phys. Rev. Lett. 60, 1218 (1988)
[16] A. Bulgac, Phys. Rev. A 37, 4084 (1988).

[17] D. I. Fevenandez et al., J. Phys. A 25, 5751 (1992).

[18] G. J. Ni, S. Q. Chen, and Y. L. Shen, Phys. Lett. A 197, 100 (1995).

[19] J. I. Cirac and P. Zoller, Phys. Rev. Lett. 74, 4091 (1995).

[20] C. Monroe, D. Leibfried, B. E. King, D. M. Meekhof, W. M. Itano, and D. J. Wineland, Phys. Rev. A 55, R2489 (1997).

[21] A. M. Childs and I. L. Chuang, Phys. Rev. A 63, 012306 (2001).

[22] A. Sørensen and K. Mølmer, Phys. Rev. Lett. 82, 1971 (1999); 82, 1835 (1999).

[23] In the subspace $\{|\widetilde{3}\rangle,|\widetilde{4}\rangle\}$, which is decoupled from the subspace $\{|\widetilde{1}\rangle,|\widetilde{2}\rangle\}$, the Hamiltonian Eq. (6) is recast as $\overline{\mathcal{H}}^{(j, k)}$ $=-\widetilde{\delta}|\widetilde{3}\rangle\langle\widetilde{3}|+\widetilde{\delta}| \widetilde{4}\rangle\langle\widetilde{4}|$, in the interaction picture with respect to the original free ion Hamiltonian. Here $\widetilde{\delta}$ denotes the ac Stark shift of the ion energy level induced by the off-resonance lasers. This energy shift will cause undesirable phase accumulations on the computational basis states $|10\rangle$ and $|01\rangle$ in the interaction picture. However, as proposed in Ref. [22], this phase accumulation can be cancelled out if one swaps the detunings of the two lasers at the half time of the two-bit operation, i.e., the $U_{j k}(\alpha)$ gate in Eq. (8). Notice that the swap of laser detunings does not affect the Hamiltonian (7) in the subspace $\{|\widetilde{1}\rangle,|\widetilde{2}\rangle\}$. We thus do not explicitly mention this point 
further in the two-bit operations.

[24] D. Loss and D. P. DiVincenzo, Phys. Rev. A 57, 120 (1998).

[25] A. Imamoglu, D. D. Awschalom, G. Burkard, D. P. DiVincenzo, D. Loss, M. Sherwin, and A. Small, Phys. Rev. Lett. 83, 4204 (1999).

[26] X. Q. Li and Y. J. Yan, Appl. Phys. Lett. 81, 168 (2002); e-print quant-ph/0204027.
[27] This can be achieved by setting $\delta_{1}=\delta_{2}$. In this case, the ac Stark shift would reduce or enhance the atomic level spacings, depending on whether the detuning between the laser frequency and the level spacing is positive or negative. As a result, for identical laser-atom coupling strengths, the net effect of Stark shifts does not break down the resonance condition, namely, $E_{1}-E_{2}=\omega_{L, 1}+\omega_{L, 2}$. 